TITLE:

\title{
Experimental simulation of spacecraft charging by artificial ion beam emission
}

$\operatorname{AUTHOR}(S)$ :

Funaki, I; Usui, H; Nakayama, Y; Kuninaka, H

\section{CITATION:}

Funaki, I ... [et al]. Experimental simulation of spacecraft charging by artificial ion beam emission. IEEE TRANSACTIONS ON PLASMA SCIENCE 2006, 34(5): 2031-2037

\section{ISSUE DATE:}

2006-10

URL:

http://hdl.handle.net/2433/40007

\section{RIGHT:}

(c)2006 IEEE. Personal use of this material is permitted. However, permission to reprint/republish this material for advertising or promotional purposes or for creating new collective works for resale or redistribution to servers or lists, or to reuse any copyrighted component of this work in other works must be obtained from the IEEE. 


\title{
Experimental Simulation of Spacecraft Charging by Artificial Ion Beam Emission
}

\author{
Ikkoh Funaki, Member, IEEE, Hideyuki Usui, Yoshinori Nakayama, and Hitoshi Kuninaka
}

\begin{abstract}
Charging of a small electrically floated body, equipped with an unneutralized microwave ion source, was experimentally studied in a vacuum chamber. When a xenon ion beam of $16 \mathrm{~mm}$ in diameter was released from a cubic floating body, $10 \mathrm{~cm}$ on each side, the body was negatively charged at a rate of $I_{\mathrm{b}} / C$, where $I_{\mathrm{b}}$ is the ion-beam current and $C$ is the capacitance between the floated body and the ground. For large $I_{\mathrm{b}} / C$ parameters, the body potential changes faster than the xenon ions can react. This simulation results in space-charge-induced potential oscillations, which are detected in experimental measurements and observed in numerical simulations. The time scale of the oscillation relaxation time was found to be at least several times, corresponding to the ion movements around the body. As expected, the steady-state potential of the floated body was relatively negative to the vacuumfacility ground at a value equal to the magnitude of the ion-beam voltage.
\end{abstract}

Index Terms-Ion thruster, plasma sheaths, space vehicle propulsion, spacecraft charging.

\section{INTRODUCTION}

$\mathbf{O}$ VER THE past several decades, many spacecrafts have utilized plasma-based devices for charge control and propulsion applications. For example, the international space station controls its potential by plasma contactors, which prevent the spacecraft from being negatively charged by actively emitting low-energy electrons [1]. Also, electric propulsion systems are widely used for north-south station keeping for geosynchronous satellites [2] and even as the main propulsion system for interplanetary missions [3], [4]. These devices emit large beams of energetic ions at rates of tens to thousands of milliamperes that are neutralized by electron emission at equivalent rates. The effect of these devices on spacecraft charging in the event of a neutralizer system failure should be carefully assessed to prevent any arcing that can be caused by net positive ion emission driving the spacecraft frame to large negative potentials. Here, we focus our attention on

Manuscript received December 23, 2005; revised May 15, 2006. This study was carried out as a part of "Ground Research Announcement for Space Utilization" promoted by Japan Space Forum.

I. Funaki is with the Institute of Space and Astronautical Science, Japan Aerospace Exploration Agency, 3-1-1 Yoshinodai, Sagamihara, Kanagawa 229-8510, Japan (e-mail: funaki@isas.jaxa.jp).

H. Usui is with the Research Institute for Sustainable Humanosphere, Kyoto University, Uji, Kyoto 611-0011, Japan (e-mail: usui@ rish.kyoto-u.ac.jp).

Y. Nakayama is with the National Defense Academy, Yokoshuka, Kanagawa 239-8686, Japan (e-mail: ynakayam@nda.ac.jp).

H. Kuninaka is with the Institute of Space and Astronautical Science, Japan Aerospace Exploration Agency, 3-1-1 Yoshinodai, Sagamihara, Kanagawa 229-8510, Japan (e-mail: kuninaka@isas.jaxa.jp).

Digital Object Identifier 10.1109/TPS.2006.883396 spacecraft charging by ion-beam emission. In contrast, lowenergy electron-producing plasma devices usually stabilize spacecraft potential because the plasma device potential is fixed to the space potential following the principle of the emissive probe [5].

Transient absolute charging processes of a spacecraft with ion-beam emission were studied during the SCATHA flight program [6]; however, the authors are not aware of any ground experiments that have been conducted in this area. To address this deficiency, a scale model experiment in a vacuum chamber was designed and conducted to characterize the transient spacecraft charging due to ion-beam emission.

\section{TheORETICAL PREDICTION}

To illustrate the spacecraft charging due to positive ionbeam emission and accompanying plasma responses around the spacecraft, a numerical simulation was performed using the code of Usui et al. [7]. This code was used to calculate the timedependent motion of ions (but electrons are neglected), which are allowed to experience Coulomb collisions. Chargeexchange collisions between the neutrals and the charge particles can also be incorporated to study the evolution of slow plasma ion populations produced by charge-transfer reactions that occur near the ion source. Although the charge-transfer process was not taken into account in the result presented below, it was observed to affect the dynamics of potential oscillations, and it is important to consider [7]. The code neglects the effects of magnetic field.

Fig. 1 contains simulation results of one of the charging experiments conducted in the vacuum chamber that is to be described in this paper. At the upper left, an electrically floated body is shown emitting an ion beam. In the plot at the lower left, ion trajectories are shown at an early stage of the charging experiment. The potential of the body decreases with time (as shown in the inset plot located at the top, right-hand corner of Fig. 1) as the floating body continues ejecting a net current of positively charged particles. The potential of the body continues decreasing, until most of the emitted ions are reflected back to the floating body. In the absence of vacuum-facility interactions, it is noted that the potential of the body must eventually reach a steady-state value that is relatively negative to the vacuum chamber walls and is equal in magnitude to the beam voltage.

The charging profile, that is, the transient potential profile of an electrically floated body with ion-beam emission, is affected by ion-beam characteristics, and electric capacitance of the 

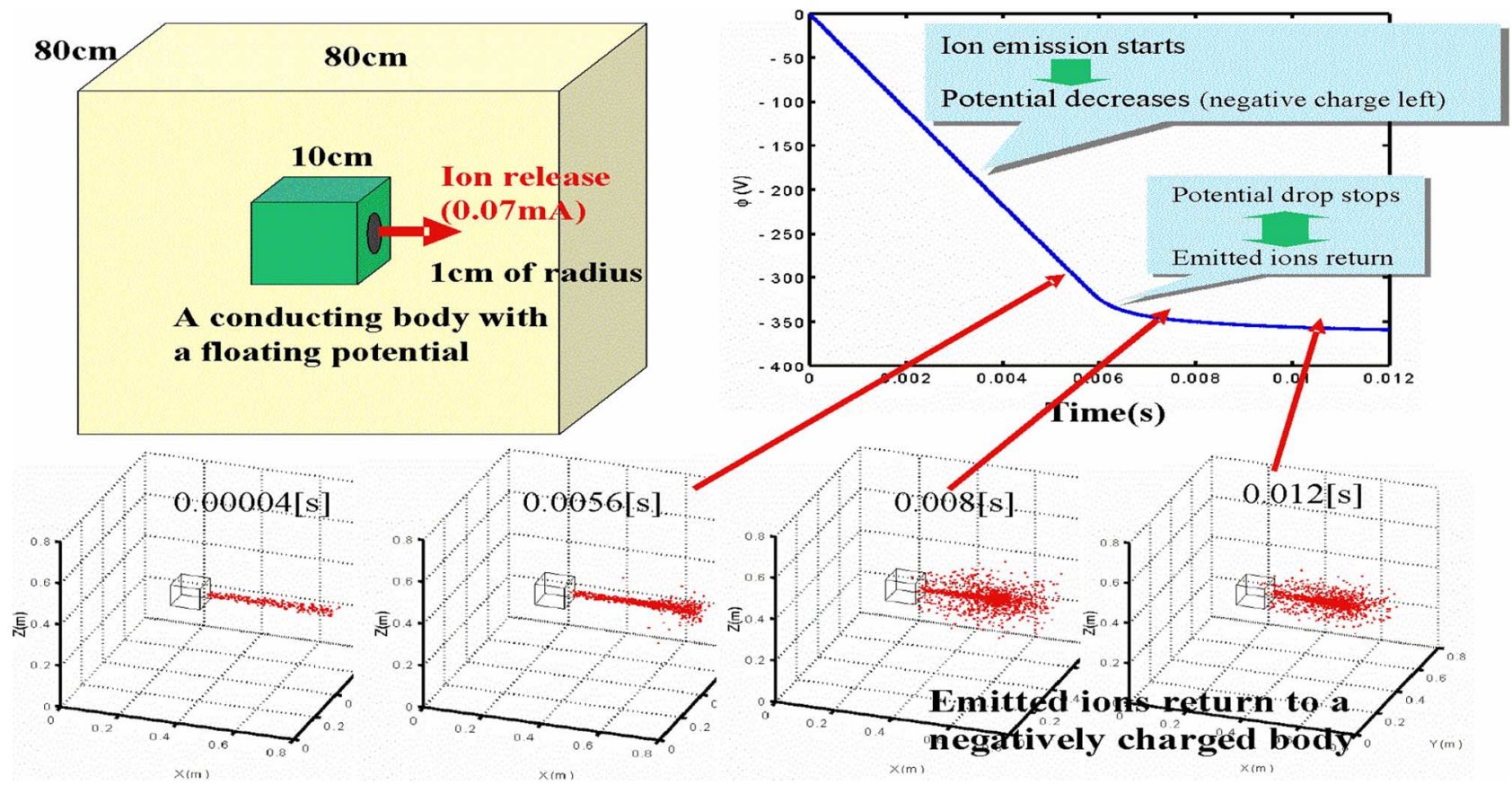

Fig. 1. Illustration of absolute charging of an electrically isolated body due to ion-beam emission; at $t=0$, a $0.07-\mathrm{mA}$ ion beam of 1 -cm radius is released (upper left figure). During the simulation, the ion beam is continuously released at a voltage of $400 \mathrm{~V}$, resulting in a negatively charged potential of the body (upper right figure). Positive ions released from the body are eventually attracted to the negatively charged body (lower figures). (Color version available online at http://ieeexplore.ieee.org.)

body as well as transient plasma responses to the change of the body potential. The effect of these parameters on charging characteristics is experimentally evaluated using the facility described in Section III below.

\section{EXPERIMENTAL APPARATUS}

We employed a $10 \mathrm{~cm} \times 10 \mathrm{~cm} \times 10 \mathrm{~cm}$ conducting body for the charging experiment, on which a 1.6-cm diameter microwave ion source is located. As shown in Fig. 2(a), the discharge chamber features a very small $1.8-\mathrm{cm}$ inner diameter. This discharge chamber was originally designed as a neutralizer for the ion thruster system onboard the asteroid explorer HAYABUSA spacecraft [3], [8]. The xenon propellant is introduced at a mass flow rate of $0.05-0.2 \mathrm{sccm}$. Microwave power of $4.2 \mathrm{GHz}$ is fed through a coaxial line terminated with an L-shaped antenna, whose tip is inserted into a magnetic field formed by front and back pole pieces and block magnets. The strong magnetic field above the electron-cyclotron-resonance condition enables easy startup of the small ion source. For example, after introducing Xe into the discharge chamber, only $5 \mathrm{~W}$ of microwave power was required to ignite the plasma. The microwave cavity was tuned by a stub located outside the chamber to minimize reflected power. The grid system to extract xenon ions is composed of three stainless-steel grids (0.3-mm thickness for the screen grid, 0.6-mm thickness for the accelerator grid, and $0.3-\mathrm{mm}$ thickness for the decelerator grid), a mica sheet with $0.25-\mathrm{mm}$ thickness, and six alumina ceramic screws. The mica sheet and ceramic screws are used to electrically isolate and set the separation distance between the grids. The screen, accelerator, and decelerator grids contain 31 apertures that are 2, 1.2, and $1.6 \mathrm{~mm}$ in diameter, respectively. The hole-to-hole spacing was $2.8 \mathrm{~mm}$.
The test configuration of the body is described in Fig. 2(b) and (c). The microwave ion source is set in a mesh case that was electrically attached to the spacecraft model body and the decelerator grid. To extract an ion beam, the screen grid is biased up to $500 \mathrm{~V}$ relative to the mesh case, and the accelerator grid is biased to $-200 \mathrm{~V}$. An optional electron emitter (thoriated tungsten filament) was used to check that the grid system was working fine without electron backstreaming toward the screen grid. The mesh case and the spacecraft model body can be electrically floated from the vacuum chamber as shown in Fig. 2(d). The experiments were conducted in a vacuum chamber, $0.8 \mathrm{~m}$ in diameter and $0.8 \mathrm{~m}$ in length, which was evacuated by turbo molecular pumps that were capable of maintaining the pressure below $10^{-5}$ torr during ion-source operation. The power supplies used to operate the ion source are located outside the vacuum chamber.

Performance and operational conditions of the ion source are summarized in Table I. One has to pay attention to the definition and value of $C$ (capacitance) in this table because two kinds of capacitance are considered herein; one is the capacitance which arises between the body and the vacuum chamber $\left(C_{\text {org }}\right)$. Capacitance due to cable connections between the body and the power supply units $\left(C_{\text {cable }}\right)$ is also included in $C_{\text {org }}$. Another one is purely external capacitance $C_{\text {add }}$ which is created by placing a capacitor between the body and the ground as shown in Fig. 2(d). Both $C_{\text {org }}$ and $C_{\text {add }}$ were measured using a capacitance meter prior to the experiment, and $C=C_{\text {org }}+C_{\text {add }}$. The value of $C_{\text {org }}$ was $0.6 \mathrm{nF}$, which included $C_{\text {cable }}$, the capacitances of sheath $\left(C_{\text {sheath }}\right)$, and the capacitance between the chamber wall and the body $\left(C_{\text {vacuum }}\right)$. Since $C_{\text {org }}=C_{\text {cable }}$ $+1 /\left(1 / C_{\text {sheath }}+1 / C_{\text {vacuum }}\right)$ and $C_{\text {vacuum }} \sim 0$ when the sheathto-wall capacitance is shorted out by currents flowing the plasma, $C_{\text {org }} \sim C_{\text {cable. }}$. 


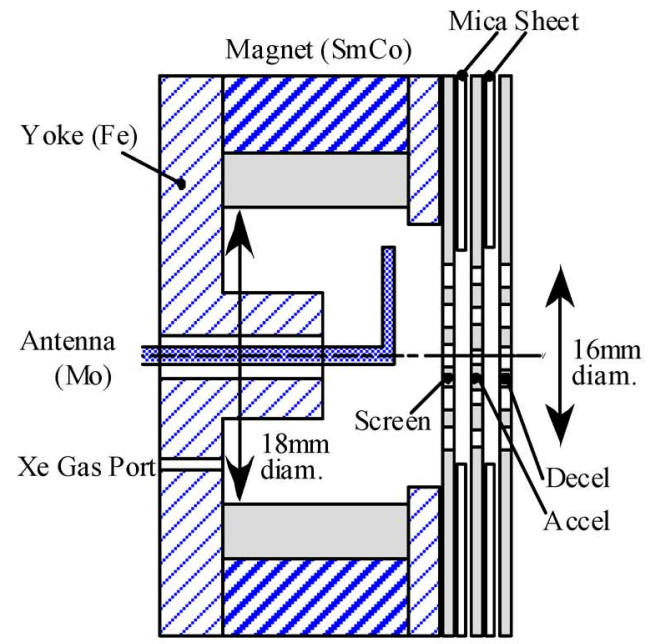

(a)

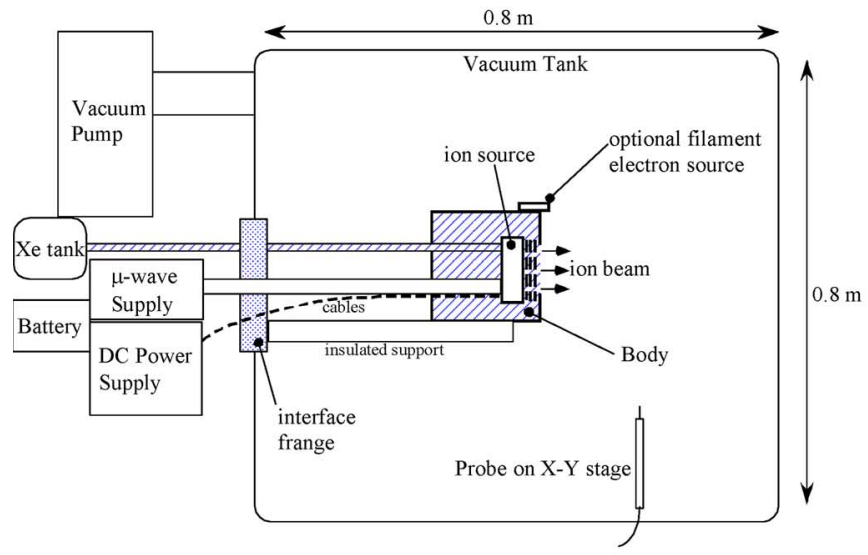

(c)

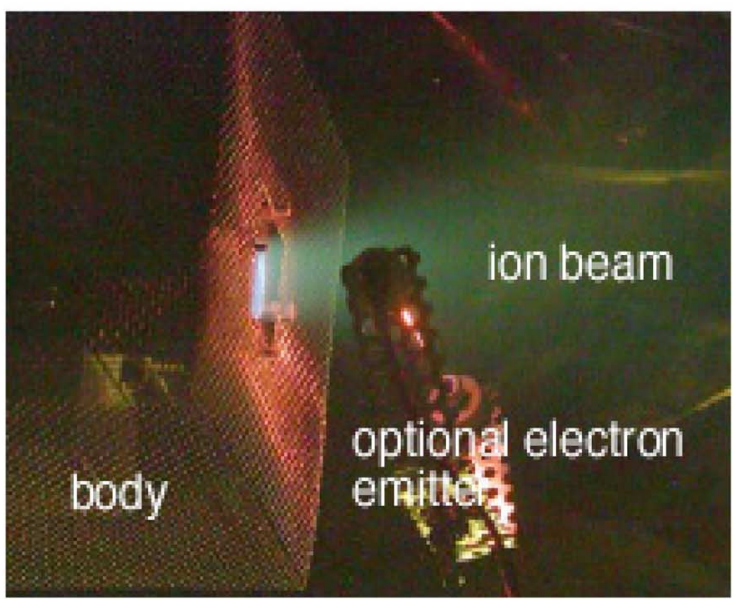

(b)

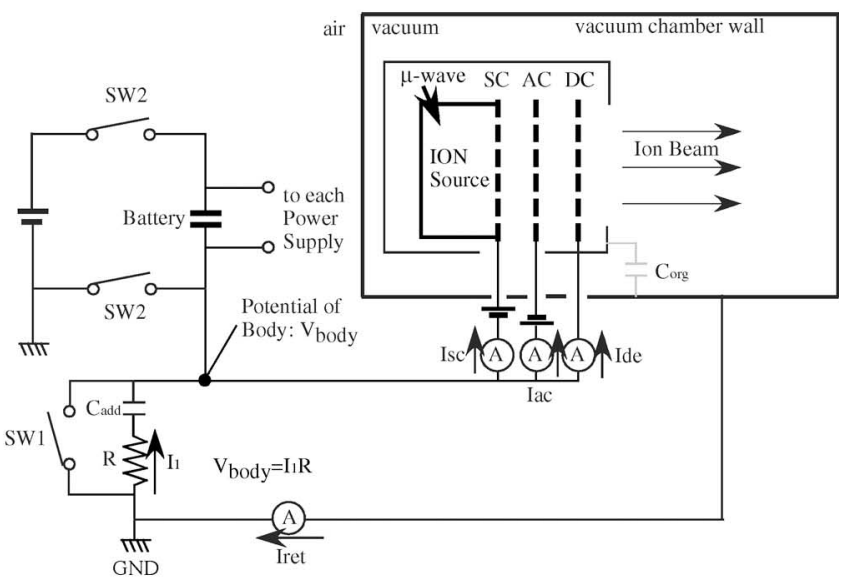

(d)

Fig. 2. Experimental setup. (a) Ion source. (b) Ion emitting body in operation. (c) Setup of scale model body in vacuum chamber. d) Electrical connections. (Color version available online at http://ieeexplore.ieee.org.)

TABLE I

OPERATIONAL PARAMETERS OF ION SOURCE AND BODY

\begin{tabular}{ll}
\hline Beam Diameter & $16 \mathrm{~mm}$ \\
Screen Voltage & 300 to $500 \mathrm{~V}$ \\
$\begin{array}{l}\text { Beam Current } \\
\text { (depending on screen voltage) }\end{array}$ & 0.5 to $1.2 \mathrm{~mA}$ \\
Xe Mass Flow Rate & $0.05 \mathrm{sccm}$ \\
Vacuum & $10^{-5} \mathrm{Torr}$ \\
Capacitance $\left(\mathrm{C}=\mathrm{C}_{\mathrm{org}}+\mathrm{C}_{\mathrm{add}}\right)$ & 0.6 to $50 \mathrm{nF}$ \\
\hline
\end{tabular}

The charging experiment was conducted using the procedure listed below. First, an ion beam was extracted from the ion source with the floated body connected to the chamber by closing SW1. During the ion-source operation, the screen current $\left(I_{\mathrm{sc}}\right)$, accelerator current $\left(I_{\mathrm{ac}}\right)$, decelerator current $\left(I_{\mathrm{de}}\right)$, and return current $\left(I_{\text {ret }}\right)$ were monitored as indicated in Fig. 2(d). Next, SW1 was opened, and the body potential and currents listed above were monitored using an oscilloscope. Note that all charging experiments were conducted with SW2 open, and that, SW2 was only closed during periods between experiments to recharge the batteries used to operate the screen and the accelerator power supplies.

\section{EXPERIMENTAL RESUlTS AND DisCUSSION}

Neutral xenon atoms flow from the ion source along with the ions that are being extracted. Some of the neutral xenon atoms experience charge-exchange collisions with fast ions, resulting in the production of a plasma containing low-energy ions that remains near the body. Typical plasma parameters in the beam and around the body were measured by Langmuir probes and Faraday cups and are listed in Table II [9]. It was observed that the temperatures of our microwave plasmas were usually high [10]. Since the Debye length is much smaller than the size of the body, when the floated body is negatively charged, the potential of the body is shielded by the ambient plasma. Due to this Debye shielding effect, the effect of chamber wall potential is also shielded; hence, a charging experiment in a small vacuum chamber is plausible.

A transient charging example in Fig. 3 shows the body potential evolution for $C=0.63 \mathrm{nF}$ and $I_{\mathrm{b}}=1.1 \mathrm{~mA}$. In Fig. 3, when SW1 is opened at $3.1 \mathrm{~s}$, the ion-beam emitting body is electrically floated and the body experiences a drastic change of its potential. Once the body potential drops below $-350 \mathrm{~V}$, the body potential rate change decreases significantly. After $30 \mathrm{~s}$, the body reaches an equilibrium potential of about $-460 \mathrm{~V}$ 
TABLE II

TYPICAL PLASMA PARAMETERS

\begin{tabular}{ll}
\hline Around the body & \\
Plasma number density & $10^{12} \mathrm{~m}^{-3}$ \\
Electron temperature & $5 \mathrm{eV}$ \\
Debye length & $0.017 \mathrm{~m}$ \\
Ion plasma frequency & $10^{5} \mathrm{rad} / \mathrm{s}$ \\
Langmuir and Blodgett sheath length & $0.35 \mathrm{~m}$ \\
$\quad$ & \\
$\quad$ (when body potential is $300 \mathrm{~V})$ & \\
Near the vacuum chamber wall & $10^{10} \mathrm{~m}^{-3}$ \\
Plasma number density & $5 \mathrm{eV}$ \\
Electron temperature & $0.17 \mathrm{~m}$ \\
Debye length & \\
In the beam & $10^{13}$ to $10^{14} \mathrm{~m}^{-3}$ \\
Plasma number density & $5 \mathrm{eV}$ \\
Electron temperature & 5.2 to $1.7 \mathrm{~mm}$ \\
Debye length &
\end{tabular}

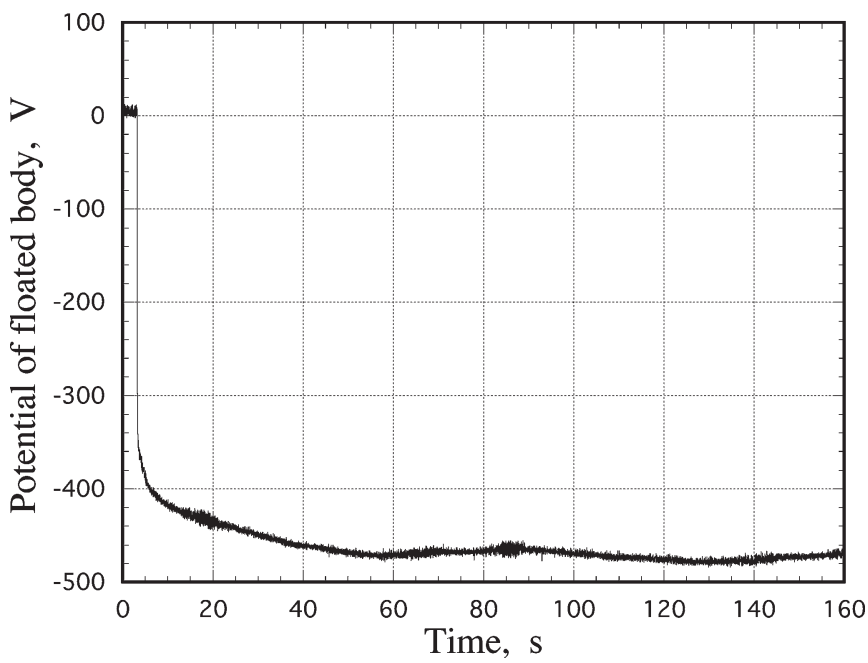

Fig. 3. Typical charging profile of the ion-beam emitting body $\left(V_{\mathrm{sc}}=500 \mathrm{~V}\right.$, $I_{\mathrm{b}}=1.1 \mathrm{~mA}, C=0.63 \mathrm{nF}$ ).

with persistent oscillations of $\pm 20 \mathrm{~V}$. What happens in this very long-term oscillation is discussed below.

The body potential and current waveforms recorded during the charging process are plotted in Fig. 4. Prior to the current measurement, Kirchhoff's Law is checked to validate the current measurements. When the body is grounded (i.e., when $\mathrm{SW} 1$ is closed), the ion-beam current $I_{\mathrm{b}}$ balances with $I_{\mathrm{ret}}$ as

$$
I_{\mathrm{b}}=I_{\mathrm{sc}}-I_{\mathrm{ac}}-I_{\mathrm{de}}=I_{\mathrm{ret}}
$$

which assures that the current flow is conserved. In Fig. 4, even when the body is floated and the potential drastically changes at $t=0.008 \mathrm{~s}, I_{\mathrm{sc}}$ is not affected; this means that the ion beam extracted from the screen grid is constant during the charging process. After this initial charging period, the accelerator current $I_{\mathrm{ac}}$ and the body current $I_{\mathrm{de}}$ begin to change until $t=0.009 \mathrm{~s}$. At this time, ions emitted from the screen grid are incident on either the accelerator grid or the body, forming a current loop between the discharge chamber and the body. The ion-beam current at this steady stage is almost zero, so that $I_{\mathrm{b}}=I_{\text {ret }} \sim 0$, and almost no outgoing ion current is observed.

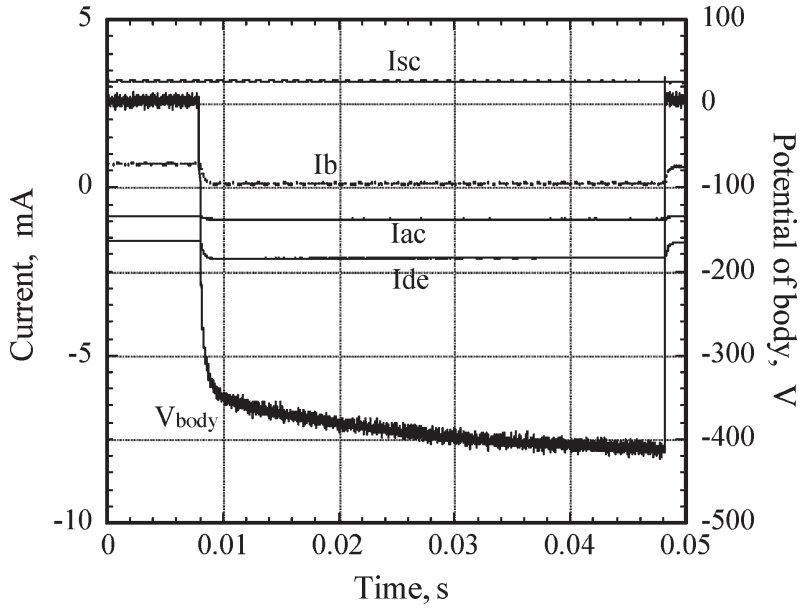

Fig. 4. Typical current profile $\left(V_{\mathrm{sc}}=500 \mathrm{~V}, I_{\mathrm{b}}=1.1 \mathrm{~mA}, C=0.63 \mathrm{nF}\right)$; $I_{\mathrm{sc}}$ is the screen current, $I_{\mathrm{ac}}$ is the accelerator current, $I_{\mathrm{de}}$ is the decelerator current, and $I_{\mathrm{b}}$ is the ion-beam current.

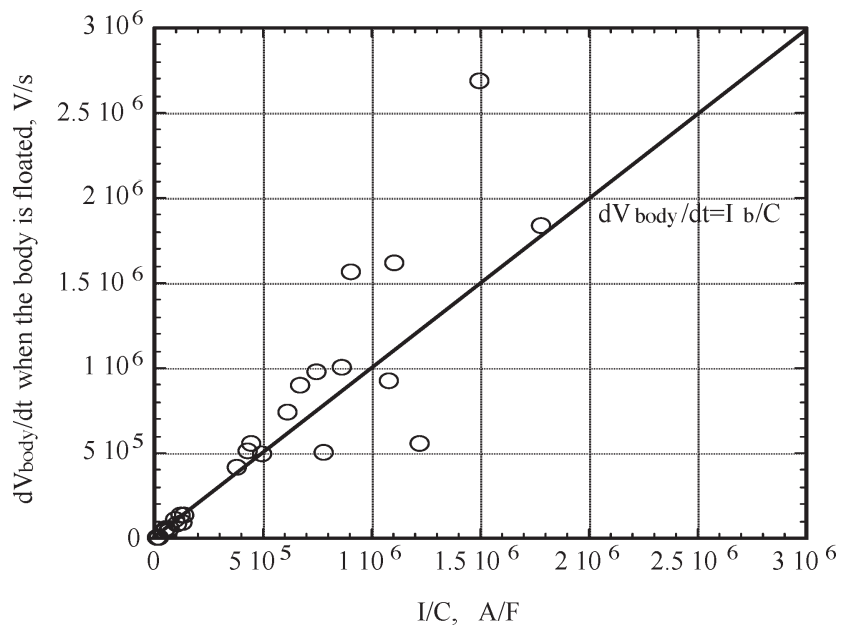

Fig. 5. $d V_{\text {body }} / d t$ versus $I_{\mathrm{b}} / C$ : Transient responses of the floating body potential $\left(d V_{\mathrm{body}} / d t\right)$ are plotted against $I_{\mathrm{b}} / C$ at the time when the body is floated.

Finally, at $t=0.048 \mathrm{~s}$, the body is grounded. Although the body potential is suddenly returned to zero, $I_{\mathrm{ac}}$ and $I_{\mathrm{de}}$ recover to original values rather slowly. Slow changes of $I_{\mathrm{ac}}$ and $I_{\mathrm{de}}$ are inferred to indicate that the ion motion responds to the potential change of the body rather slowly.

If the above charging experiment in Fig. 4 was continued, the long-term potential change in Fig. 3 would be obtained. The discrepancy between the magnitude of the equilibrium potential $(460 \mathrm{~V})$ and the beam voltage $(500 \mathrm{~V})$ is attributed to the secondary electron emission when the returning ions impinge on either the accelerator grid or the decelerator grid. If the secondary electrons emitted from the accelerator grid hit the ambient neutral particles, it will produce a plasma and enhance the ions impinging on the accelerator grid. To prohibit inducing arcing, the neutral density (or equivalently, the pressure) in the vacuum chamber was appropriately selected.

The secondary emitted electrons also play an important role in periodic oscillations of the body potential. A slight change in 


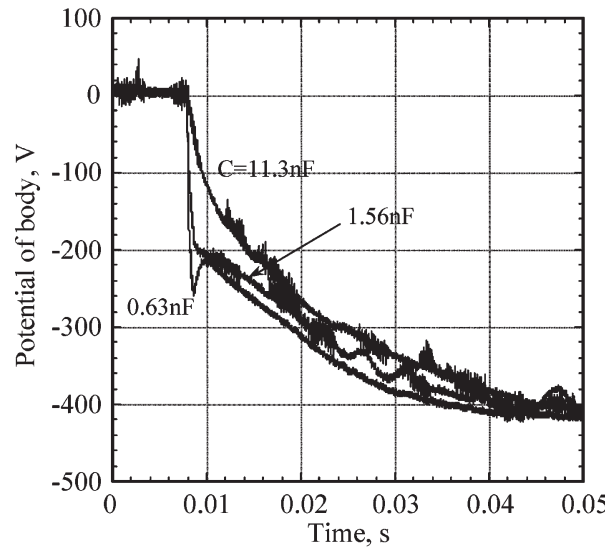

(a)

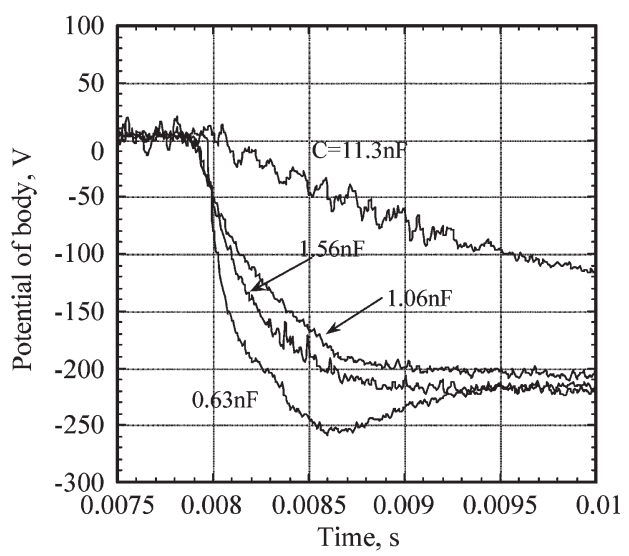

(b)

Fig. 6. Charging profiles in the case of $I_{\mathrm{b}}=1.1 \mathrm{~mA}$ and $V_{\mathrm{sc}}=500 \mathrm{~V}$ for (a) various capacitance (0.63 to $11.3 \mathrm{nF}$ ) and (b) close-up view.

the body potential can increase the ion-impinging and electronemitting currents, hence resulting in a large potential change of the body; this is because, the electron emission as well as the ion-impinging current to the body are increased even when the body potential approaches an equilibrium potential. This reasonably explains the long-term oscillation in Fig. 3.

The capacitance of the body relative to the vacuum chamber has a first-order effect on the charging processes, and tests were performed by adding additional capacitance between the body and ground. The time scale of charging can be controlled by the following equation:

$$
\frac{d V_{\mathrm{body}}}{d t}=\frac{I_{\mathrm{b}}}{C}
$$

In Fig. 5, the rate of potential change $d V_{\text {body }} / d t$ for various $I_{\mathrm{b}} / C$ parameters is plotted. Here, each " $d V_{\mathrm{body}} / d t=I_{\mathrm{b}} / C$ " line corresponds to the case where the charging time scale is strictly determined by (2). However, for larger $I_{\mathrm{b}} / C$ values, the values of $d V_{\text {body }} / d t$ deviate from (2), which may be attributed to the ambiguity in $C$ in particular when additional capacitance is not used.

In Fig. 6, the time histories of the body potential are plotted for various values of capacitance $C$. For large $I_{\mathrm{b}} / C$ parameters, a steep drop of the potential was observed at the beginning of the charging process, which sometimes causes potential overshoot as in the case of $C=0.63 \mathrm{nF}$. The potential change for all of these cases is quick; hence, it is expected that ions cannot respond to this fast potential variation of the body. The time scale of the potential variation $d t \sim 0.1 \mathrm{~ms}$ is slower than the time based on the ion plasma frequency, $2 \pi / \omega_{\mathrm{pi}} \sim 0.06 \mathrm{~ms}$ for $10^{12} \mathrm{~m}^{-3}$, where $\omega_{\mathrm{pi}}$ is the ion plasma frequency. Hence, the characteristic time is expected to be the ion acoustic time scale.

During the charging process in Figs. 3 and 6, strong spacecharge-limited ion flow is expected in front of the grid of the ion source. [6] At short times, the presence of energetic ions can increase the space-charge-induced potential blow up that occurs in front of the grid. This region of increased space potential is called herein a potential hill and denoted as $V_{\mathrm{VA}}$ in Fig. 7(a). If the potential of the body is fixed, the space-charge-limited flow would result in a potential maximum $V_{\mathrm{VA}}$ as $V_{\mathrm{VA}}-V_{\text {body }}=$

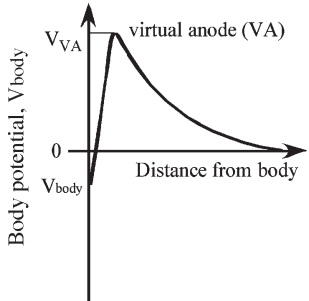

(a)

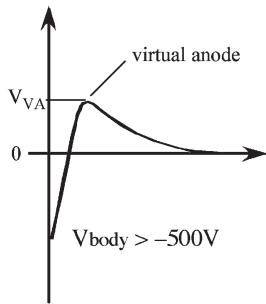

(b)

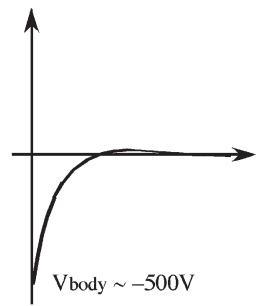

(c)
Fig. 7. Schematics of virtual anode formation in front of the ion source for: (a) After the body is floated and VA develops, (b) during the charging process; and (c) after the body is completely charged and VA almost disappears.

$V_{\text {sc }}$ [11]. The Child-Langmuir Law for space-charged-limited current density is

$$
j=A V_{\mathrm{VA}}^{3 / 2}
$$

where $A$ is a constant. This equation governs how much ion current can escape from the ion source, and hence controls the charging rate. As time increases, $V_{\text {body }}$ drops and the potential hill $V_{\text {VA }}$ gradually decreases [Fig. 7(b)] and eventually disappears [Fig. 7(c)]. [9] This situation will apply to our charging experiment when the charging rate is slow; that is, for $d t \gg 2 \pi / \omega_{\mathrm{pi}}$. If the rate of charging is large and the ion time scale is large, the ions cannot respond to the potential change of the body and the above discussion will break down. This situation would most likely lead to space-charge-induced oscillations.

A close-up view of oscillations observed during the charging process is shown in Fig. 6(b). Three kinds of frequencies were identified: one is very slow at 0.1 to $10 \mathrm{~Hz}$, which is caused by slow ions near the potential hill of the virtual anode. These slow oscillations are mostly evident in Fig. 3. Potential oscillations are also observed at higher frequencies of 1 and $300 \mathrm{kHz}$. The $300-\mathrm{kHz}$ oscillation corresponds to the ion acoustic frequency in the artificially created charge-exchange plasma. The intermediate frequency of $1 \mathrm{kHz}$ is believed to correspond to the ion movement between the ion source and the potential hill.

It is important to discuss how the above transient charging tests in the ground chamber will apply to a realistic spacecraft. 


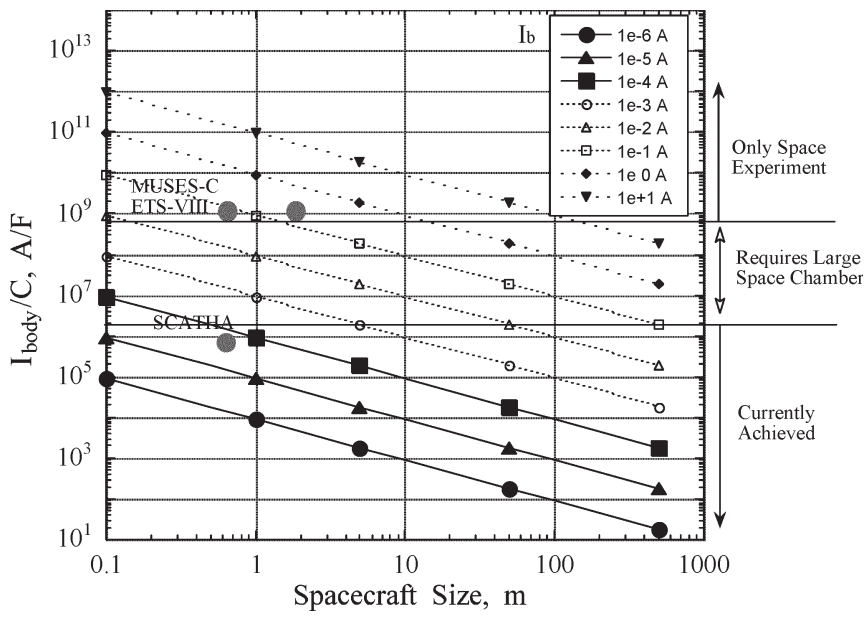

Fig. 8. Scaling considerations.

To simulate spacecraft charging in space, the parameters in Tables I and II (i.e., the body size, the ion-beam current, the ionbeam voltage, the ambient plasma, and, in particular, the $I_{\mathrm{b}} / C$ parameter) should be carefully considered. The capacitance of a spacecraft is roughly approximated by its size, and the $I_{\mathrm{b}} / C$ parameter is determined by the beam current $I_{\mathrm{b}}$ that is available. For example, many commercial satellites are equipped with 0.1 to 3 A-class ion thrusters for north-south station keeping and orbit-raising applications. In Fig. 8, the $I_{\mathrm{b}} / C$ parameters are plotted against the spacecraft size for different beam current levels, with the characteristics of some typical spacecrafts. The time scales currently achieved in our present experiment are rather slow; hence, we are planning to make a device that can simulate charging phenomena which occur in a very short time scale, that is, two orders of magnitude faster than our current experimental capability.

\section{SUMMARY}

The transient charging processes of an electrically floated body with ion-beam emission were experimentally studied. The $10-\mathrm{cm}$ cubic body was located in a vacuum chamber with a small 16-mm beam diameter microwave ion source, which was operated at a beam voltage of up to $V_{\mathrm{sc}}=500 \mathrm{~V}$ and at an ionbeam current $I_{\mathrm{b}} \leq 1.1 \mathrm{~mA}$. The system was used to simulate the spacecraft charging when complete failure of the neutralization system occurs. When electrically floated, the body was initially negatively charged following $d V_{\text {body }} / d t=I_{\mathrm{b}} / C$. For large $I_{\mathrm{b}} / C$ parameters, $V_{\text {body }}$ linearly decreased during the initial charging process of the body until the emitted ions were attracted back to the negatively charged body. After this initial and quick charging phase, the body potential only gradually changed until it obtained an equilibrium state. Some potential oscillations were observed during the fast and slow charging processes which were attributed to ion motions nearby the body. In all experiments, the body potential was observed to acquire a quasi-steady potential that was negative of the vacuum facility and equivalent in the magnitude to the ion-beam voltage. In this situation, an ion sheath is expected to be formed around the body. At the positive edge of this ion sheath, all of the ions released from the body lose their forward momentum, and most of them will return back to the body. However, a small amount of charge-exchange ion production is predicted to occur, and these low-energy ions remain near the sheath edge for relatively long periods of time. Collective motion of these trapped ions is suspected to cause long-term oscillations of the body potential that have been observed in our experiments.

\section{REFERENCES}

[1] M. R. Carruth et al., "ISS and space environment interactions without operating plasma contactor," presented at the 39th AIAA Aerospace Science Meeting and Exhibit, Reno, NV, 2001, AIAA-2001-0401.

[2] D. A. Lichtin, "An overview of electric propulsion activities in US industry," presented at the 41st AIAA/ASME/SAE/ASEE Joint Propulsion Conf. and Exhibit, Tucson, AZ, Jul. 2005, AIAA-2005-3532.

[3] H. Kuninaka, K. Nishiyama, I. Funaki, Y. Shimizu, T. Yamada, and J. Kawaguchi, "Assessment of Plasma Interactions and Flight Status of the HAYABUSA Asteroid Explorer Propelled by Microwave Discharge Ion Engines," IEEE Trans. Plasma Sci., vol. 34, no. 5, pp. 2125-2132, Oct. 2006.

[4] J. Brophy, M. Marcucci, J. Gates, C. Garner, B. Nakazono, and G. Ganapathi, "Status of the DAWN ion propulsion system," presented at the 40th AIAA/ASME/SAE/ASEE Joint Propulsion Conf. and Exhibit, Fort Lauderdale, FL, Jul. 2004, AIAA-2004-3433.

[5] F. F. Chen, "Electric probes," in Plasma Diagnostic Techniques. New York: Academic, 1965, ch. 4.

[6] S. T. Lai, "An overview of electron and ion beam effects in charging and discharging of spacecraft," IEEE Trans. Nucl. Sci., vol. 36, no. 6, pp. 2027-2032, Dec. 1989.

[7] H. Usui, H. Matsumoto, M. Yasugi, and Y. Omura, "PIC simulations of spacecraft charging and its neutralization process by plasma emission," Advances in Space Research, vol. 34, no. 11, pp. 2437-2440, 2004.

[8] I. Funaki and H. Kuninaka, "Overdense plasma production in a low-power microwave discharge electron source," Jpn. J. Appl. Phys. 1, Regul. Rap. Short Notes, vol. 40, no. 4A, pp. 2495-2500, 2001.

[9] H. Kuninaka, I. Funaki, K. Nishiyama, and Y. Nakayama, "Virtual anode phenomena due to lack of neutralization on ion thrusters based on MUSES-C program," presented at the 37th AIAA/ASME/SAE/ASEE Joint Propulsion Conf. and Exhibition, Salt Lake City, UT, Jul. 2001, AIAA2001-3785.

[10] I. Funaki, H. Kuninaka, and K. Toki, "Plasma characterization of a 10-cm diameter microwave discharge ion thruster," J. Propuls. Power, vol. 20, no. 4, pp. 718-727, 2004.

[11] J. Wang and S. T. Lai, "Virtual anode in ion beam emissions in space: Numerical simulations," J. Spacecr. Rockets, vol. 34, no. 6, pp. 829-836, 1997.

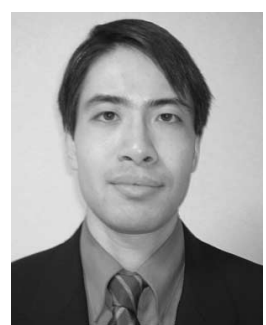

Ikkoh Funaki (M'00) received the B.S. degree from the Department of Aeronautics, Kyoto University, Kyoto, Japan, in 1990 and the M.S. and Ph.D. degrees from the Department of Aeronautics and Astronautics, University of Tokyo, Tokyo, Japan, in 1992 and 1995, respectively.

During 1995 and 2001, he was a part-time Lecturer, then a Research Associate with the Institute of Space and Astronautical Science, Kanagawa, Japan, where he engaged himself in developing the microwave discharge ion engines. In 2001, he became a Lecturer with University of Tsukuba, Japan. In 2003, he joined the Department of Space Transportation Engineering, Institute of Space and Astronautical Science, Japan Aerospace Exploration Agency, Kanagawa, Japan, where he is an Associate Professor. His current research interests are in electric and other advanced spacecraft propulsion systems, plasma application in space, and space plasma physics. 


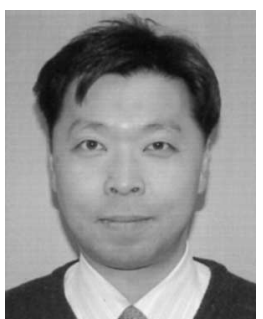

Hideyuki Usui received the B.S. degree from the Department of Electrical Engineering, Kyoto University, Kyoto, Japan, in 1986 and the M.S. and $\mathrm{Ph} . \mathrm{D}$. degrees from the Department of Electronics, Kyoto University, Kyoto, Japan, in 1989 and 1994, respectively.

In 1992, he started working as Research Assistance with Radio Science Center for Space and Atmosphere in Kyoto University, Kyoto, Japan. In 1997, he stayed as a Visiting Scientist with the Plasma Theory and Simulation Group in the University of California Berkeley. In 1999, he was promoted as the Associate Professor with Radio Science Center for Space and Atmosphere in Kyoto University, Japan. In 2000, the center was reorganized as the Research Institute for Sustainable Humanosphere in Kyoto University, Japan, where he is currently working as an Associate Professor.

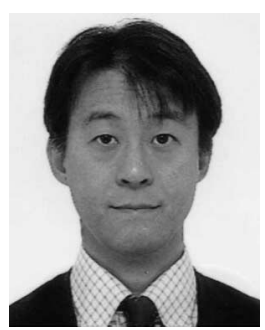

Yoshinori Nakayama received the B.S., M.S., and Ph.D. degrees from the Department of Aerospace Engineering, Tokyo Metropolitan Institute of Technology (currently, Tokyo Metropolitan University), Tokyo, Japan, in 1993, 1995, and 1999, respectively.

In 1999, he worked as a Postdoctoral Researcher with the Institute of Space and Astronautical Science, Kanagawa, Japan, promoted by Japan Society for the Promotion of Science. From 1999 to 2003, he worked as a Research Associate with the National Defense Academy, Yokoshuka, Kanagawa, Japan. From 2000 to 2001, he stayed as a Visiting Scholar with Colorado State University. He was promoted as a Lecturer with the National Defense Academy in 2003 .

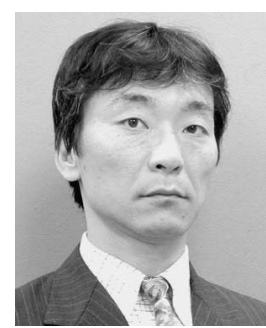

Hitoshi Kuninaka received the B.S. degree from the Department of Aeronautics, Kyoto University, Kyoto, Japan, in 1983 and the M.S. and Ph.D. degrees from the Department of Aeronautics, University of Tokyo, Tokyo, Japan, in 1985 and 1988, respectively.

He was a Research Engineer with the Institute of Space and Astronautical Science (ISAS), Japan Aerospace Exploration Agency, Sagamihara, Japan, from 1988 to 1999 . He stayed as a Visiting Scientist at the Kennedy Space Center, National Aeronautics and Space Administration, Cape Canaveral, FL, in 1996. He was promoted to Associate Professor and Full Professor with the Institute of Space and Astronautical Science, in 2000 and 2005, respectively. He also holds the post of Professor with the Department of Aeronautics and Astronautics, University of Tokyo. He researched the plasma interaction of satellites and developed electric propulsion including dc arcjets, hall thrusters, ion engines, magnetoplasmadynamic arcjets, and so on. He participated in the satellite project Space Flyer Unit from 1988 to 1996 and made it return to Earth successfully. He invented the microwave discharge ion engines for the asteroid explorer HAYABUSA. 